\title{
Anti-Mullerian hormone concentrations in individual follicular fluids within one stimulated IVF cycle resemble blood serum values
}

\author{
Michael Schenk $^{1}$ - Julia Maria Kröpfl ${ }^{2}$ - Barbara Obermayer-Pietsch ${ }^{3}$. \\ Elisabeth Feldmeier $^{1} \cdot$ Gregor Weiss $^{1}$ (i)
}

Received: 10 January 2017 / Accepted: 7 March 2017 / Published online: 21 March 2017

(C) The Author(s) 2017. This article is published with open access at Springerlink.com

\begin{abstract}
Purpose Anti-Mullerian hormone (AMH) is commonly known as the most potent marker for ovarian reserve due to its decline as female age increases. While serum AMH (sAMH) levels have been intensively investigated, there is less data regarding AMH concentrations in follicular fluid (FF), since FF has usually been designated as waste product during oocyte collection in assisted reproductive technologies. This pilot study investigated follicle AMH concentrations (fAMH) of several follicles per ovary, individually collected with the Steiner-Tan needle, and compared them to sAMH concentrations in women undergoing IVF treatment. We hypothesized that there is no difference of fAMH concentrations in individual follicles and that these concentrations resemble the sAMH value of the patient.

Methods Patients were stimulated with a gonadotropinreleasing hormone antagonist ovarian hyperstimulation protocol. On the day of oocyte retrieval, serum samples and FF from all individual follicles from one stimulated IVF cycle were collected and individually analyzed for AMH concentrations.

Results Intracyclic mean fAMH values $\left(n_{\text {follicle }}=2-14\right)$ were significantly correlated to sAHM values $(\rho=0.85, p<0.001)$
\end{abstract}

Gregor Weiss

gregor.weiss@kinderwunsch-institut.at

1 Das Kinderwunsch Institut Schenk GmbH, Am Sendergrund 11, 8143 Dobl, Austria

2 Exercise Physiology Lab, Institute of Human Movement Sciences and Sport, ETH Zürich, Winterthurerstrasse 190,

8057 Zürich, Switzerland

3 Division of Endocrinology and Diabetology, Department of Internal Medicine, Medical University of Graz, Auenbruggerplatz 15 , 8036 Graz, Austria and showed a trend to be negatively associated with age $(\rho=-0.43, p=0.06)$. Mean intrapatient fAMH concentrations differed significantly $(p<0.001)$. Furthermore, significant correlations of sAMH with individual fAMH values of the first five follicles of each patient were observed.

Conclusions In conclusion, our results clearly showed that individual fAMH concentrations reflected SAMH values and that fAMH concentrations did not significantly differ within one patient. In future studies, it will be interesting to correlate individual fAMH values to the respective embryo development and overall pregnancy outcome in order to improve IVF treatments and to refrain from embryo overproduction.

Keywords Anti-Mullerian hormone · Follicular fluid · IVF . Steiner-Tan needle

\section{Introduction}

Follicular fluid (FF) is a mixture of chemical constituents, comprising a variety of different proteins as well as growth factors, reactive oxygen species, anti-apoptotic factors, fatty acids, sugars, and hormones [1]. Among them, the AntiMullerian hormone (AMH), a homodimeric glycoprotein which belongs to the transforming growth factor- $\beta$ (TGF- $\beta$ ) family, has evolved to become one of the most prominent targets for reproductive health research [2]. Anti-Mullerian hormone is secreted primarily by granulosa cells of ovarian follicles during early stages of follicle development (pre-antral and antral follicles) [3] but has also been found in endometrial and endometriotic tissue [4]. Females are born with a defined number of primordial follicles, whose quantity and quality define the ovarian reserve [5]. Oocytes within primordial follicle rest in a dormant state of meiosis I until puberty, and until then, the granulosa cells do not secrete AMH. A recent study 
demonstrated the beginning of AMH secretion with the recruitment of the follicles [6], with maximum AMH values at the age of 15.8 years. After a plateau phase until the age of 25 years, AMH concentrations start to decrease and inversely correlate with age. This emphasizes AMH as the most potent marker for ovarian reserve in women of 25 years and older [7] among other parameters commonly used such as antral follicle count, follicle stimulating hormone (FSH), luteinizing hormone ( $\mathrm{LH})$, estradiol, or inhibin B. During the menstrual cycle serum AMH (sAMH), values remain relatively stable compared to all other hormones secreted by the ovary and can be analyzed at any day of the cycle, which is an advantage for flexible in vitro fertilization (IVF) treatment [8].

Values of sAMH have been intensively investigated [9, 10]; however, there is less data regarding the behavior of AMH concentrations in FF (fAMH), since FF has usually been designated as waste product during oocyte collection for IVF treatment. Recent studies revealed the importance of FF in oocyte development [11], providing information on follicular growth, oocyte quality, and fertilization. However, in studies to date, FFs have either been pooled [12] or solely the dominant follicle was examined [13]. Information on individual fAMH concentrations within one stimulated IVF cycle is still not available, although it is tempting to speculate that individual fAMH could be a potential predictor of fertilization success in IVF treatments, since it had already been found to correlate with the respective embryo and IVF outcome, at least regarding the FF of the dominant follicle [14]. Due to different study designs and methods used (dominant or pooled follicle analysis), it is hardly possible to compare results and draw any conclusions. Comparing all individual fAMH values with the respective embryo and IVF outcome, however, has not yet been done and would emphasize fAMH even more as potent biomarker in IVF treatment by facilitating and complementing the embryo selection process.

In this pilot study, we demonstrated that all mature follicles during a stimulated IVF cycle could be aspirated individually with the Steiner-Tan needle [15] for fAMH analysis. We hypothesized that there was no difference between fAMH concentrations of individual follicles within one patient and that these concentrations resembled the respective sAMH value of the patient.

\section{Material and methods}

\section{Study design}

Blood serum $(n=17)$ and follicular fluid samples $(n=20)$ of female volunteers, age 26-43, undergoing IVF treatment were prospectively collected and analyzed for AMH concentrations if they met the following criteria: (1) both ovaries present, (2) BMI between 19 and 30, (3) adequate visualization of ovaries in transvaginal ultrasound scan, (4) written informed consent of the patients, and (5) stimulation with gonadotropinreleasing hormone $(\mathrm{GnRH})$ antagonist protocol. Samples were collected at the Kinderwunsch Institut Dobl, Austria, between 2014 and 2015. Informed consent was obtained from each woman with approval of the ethical committee of the Medical University of Graz (approval number 20-492 ex08/09).

\section{Ovarian hyperstimulation protocol}

All women included for the study underwent GnRH antagonist protocol controlled ovarian hyperstimulation. Patients received recombinant human follicle stimulating hormone (Puregon; MSD Sharp \& Dohme GMBH) for 5 days with doses according to age, weight, sAMH, and hormonal status $[16,17]$. Trans-vaginal sonography was performed after 5 days and on the day of oocyte retrieval. Ultrasonographical measurement was performed using a RIC 5-9-D 4D intravaginal probe of a GE Voluson E8 BT09 ultrasound machine (both from GE Healthcare Austria GmbH). GnRH antagonist (Cetrotide, Merck KGaA) was injected to avoid premature ovulation. Triggering was initiated $35 \mathrm{~h}$ before the punction, administered with 5000-10,000 IU human chorionic gonadotropin (hCG) subcutaneously (Pregnyl, N.V. Organon), with dose according to body weight of the patient [16].

\section{Oocyte and follicular fluid retrieval}

Oocyte retrieval was performed under sedation (Propofol, Fresenius Kabi Austria GmbH; Rapifen, Janssen-Cilag Pharma $\mathrm{GmbH}$ ). Every follicle larger than $10 \mathrm{~mm}$ in diameter was aspirated and flushed (Flushing medium GM501 Flush; Gynemed Medizinprodukte $\mathrm{GmbH} \&$ Co.KG) under transvaginal ultrasound guidance (GE Healthcare Austria $\mathrm{GmbH})$ with a Steiner-Tan needle 17 gauge and a Steiner flush/valve (IVFETFLEX.com HandelsgmbH \& Co KG) [15]. Follicles closest to the vagina were aspirated first. Follicular fluid was examined for oocytes under constant conditions of $37{ }^{\circ} \mathrm{C}$ in an IVF workstation L24E with heating stage (K-SYSTEMS Kivex Biotec A/S) and was subsequently stored at $4{ }^{\circ} \mathrm{C}$ for further hormonal analysis. The flushing volumes were examined for oocytes and discarded afterwards. The method of collection and storage of FF as well as other body liquids within the frame of IVF (blood serum, cumulus cells, seminal plasma, embryo culture supernatant) was previously described by Schenk et al. [18], providing comprehensive information on laboratory procedures and sampling techniques to enable the comparability of future studies in the field of reproductive health research. 


\section{Blood serum collection}

Blood serum was collected on day of oocyte retrieval. At least $4 \mathrm{ml}$ of blood was taken by venipuncture into an 8 -ml vacuette tube with serum separator (Greiner Bio-One International $\mathrm{GmbH})$. Samples were centrifuged at $1800 \mathrm{~g}$ for $10 \mathrm{~min}$, and serum supernatant was collected and stored at $4{ }^{\circ} \mathrm{C}$ in $15-\mathrm{ml}$ tubes (VWR International $\mathrm{GmbH}$ ) for subsequent $\mathrm{AMH}$ analysis.

\section{Quantitative analysis of AMH}

Serum and follicular fluid AMH concentrations were determined using electrochemiluminescence immunoassay (ECLIA) for quantitative determination (Cobas-e411 analyzer, Roche Diagnostics $\mathrm{GmbH}$ ). The analysis is fully automated with a mean intra-assay coefficient of variability $(\mathrm{CV})$ of $1.34 \%$ and a mean inter-assay CV of $3.84 \%$ for sAMH according to the manufacturer's data sheet. Follicular fluid was centrifuged $10 \mathrm{~min}$ at $3000 \mathrm{~g}$ before measurement. Samples were analyzed according to the manufacturer's instructions in a measurement range of $0.01-23 \mathrm{ng} / \mathrm{ml}$. For validation of parallelism [19] of the obtained fAMH results, samples were diluted $1: 2,1: 5,1: 10,1: 20,1: 50$, and 1:100 in buffer and reanalyzed.

\section{Statistical analysis}

Data are presented as individual or mean values. All variables were tested for normal distribution with the KolmogorovSmirnov test. Depending on variables' distributions, Pearson's or Spearman's rank correlation analysis was used to detect associations between continuous variables. An a priori power analysis revealed a necessary sample size of $n=17$ in order to detect any significant relationship (effect size $=0.7$, alpha $=0.05,1$-beta $=0.92$ ). Differences between sAMH and intra-individual fAMH values of the first five follicles (median number of follicles in this patient cohort) were investigated by repeated measures ANOVA with Bonferroni post hoc corrections. Comparison of mean fAMH values of all follicles between patients was done by one-way ANOVA with Bonferroni post hoc corrections. A $p$ value $<0.05$ was considered as significant.

\section{Results}

\section{Patients' characteristics}

A total of 20 patients (age $35.8 \pm 5.5$ years; BMI $22.1 \pm 2.4 \mathrm{~kg}$ / $\mathrm{m}^{2}$ ) undergoing IVF treatment between 2014 and 2015 were analyzed (Table 1). The number of aspirated follicles ranged
Table 1 Individual patient characteristics

\begin{tabular}{llll}
\hline Patients ID & Age & BMI & $n_{\text {follicle }}$ \\
\hline 1 & 35 & 23.0 & 5 \\
2 & 32 & 22.3 & 3 \\
3 & 43 & 20.8 & 3 \\
4 & 30 & 20.3 & 14 \\
5 & 26 & 21.6 & 6 \\
6 & 32 & 21.3 & 10 \\
7 & 35 & 21.7 & 5 \\
8 & 41 & 24.6 & 3 \\
9 & 39 & 19.4 & 12 \\
10 & 42 & 30.1 & 7 \\
11 & 41 & 21.3 & 5 \\
12 & 30 & 19.9 & 13 \\
13 & 43 & 20.8 & 6 \\
14 & 36 & 20.3 & 12 \\
15 & 31 & 21.6 & 4 \\
16 & 33 & 21.8 & 6 \\
17 & 41 & 24.2 & 8 \\
18 & 43 & 21.2 & 4 \\
19 & 28 & 22.9 & 2 \\
20 & 35 & 20.3 & 5 \\
\hline
\end{tabular}

$n_{\text {follicle }}$ number of follicles collected during IVF treatment

from two to 14 , according to the number of follicles matured during IVF treatment.

\section{Inter-variable relationships}

Mean fAMH values $\left(n_{\text {follicle }}=2-14\right)$ were significantly correlated to sAMH values ( $\rho=0.85, p<0.001, n=17$; Fig. 1 ). Younger patients showed by trend higher fAMH concentrations than older ones ( $\rho=-0.43, p=0.06, n=20)$, while their body mass index was not significantly associated $(p>0.05)$.

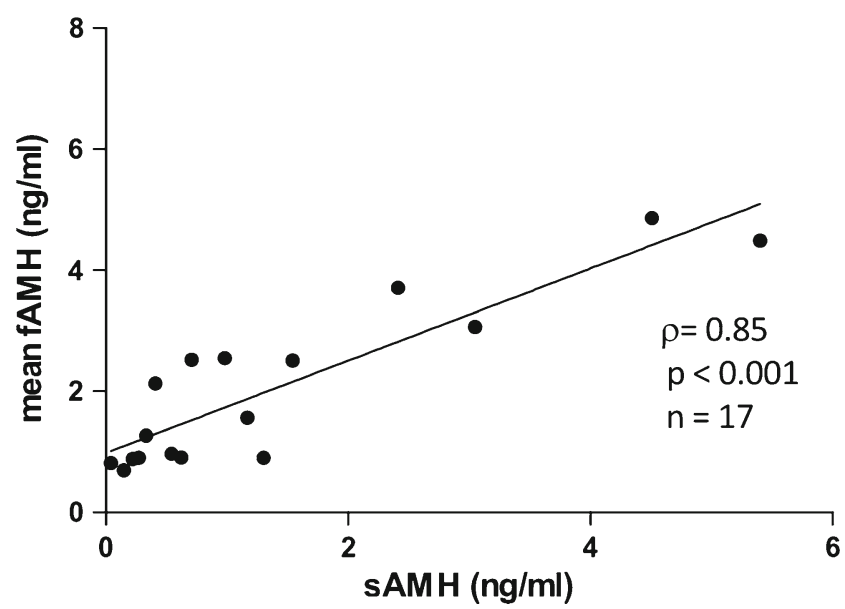

Fig. 1 Relationship of intracyclic mean fAMH values $\left(n_{\text {follikel }}=2-14\right)$ to sAHM values $(\rho=0.85)$ 
Likewise, sAMH concentrations revealed a significant relationship to age $(r=-0.53, p<0.05, n=17)$.

Furthermore, significant correlations of sAMH with individual fAMH values of the first five follicles of each patient were observed $\left(r_{1}=0.89, n=17, p<0.001 ; r_{2}=0.81, n=17\right.$, $p<0.001 ; r_{3}=0.67, n=16, p<0.01 ; r_{4}=0.66, n=14$, $p<0.05 ; r_{5}=0.70, n=12, p<0.05$ ) (Fig. 2).

\section{Quantification of AMH concentrations in blood serum and follicular fluid: intracyclic and intrapatient analysis}

It was clearly visible that $\mathrm{AMH}$ could be measured in an individual follicle, since AMH values among five different follicles of one patient were not significantly different from each other and comparable to sAMH values $(n=14, p>0.05)$.

\section{Quantification of AMH concentrations in blood serum and follicular fluid: subanalyses between patients}

Mean fAMH concentrations between patients differed significantly $\left(p<0.001, n_{\text {follicle }}\right.$ per patient $=2-14$, Fig. 3$)$.

\section{Discussion}

In the present study, we provided evidence that fAMH concentrations differed between patients but not within one stimulated IVF cycle and that individual fAMH values correlated with SAMH data of the respective patient. We demonstrated that FF can be collected from individual follicles within one stimulated cycle (intracyclic) with the Steiner-Tan needle.

In contrast to previous studies, we measured AMH concentrations of all individual follicles within one stimulated IVF cycle of patients undergoing IVF treatment and demonstrated a significant correlation between SAMH and mean/individual fAMH values within patients. This AMH data of all individual mature follicles is unique and supports other studies, which

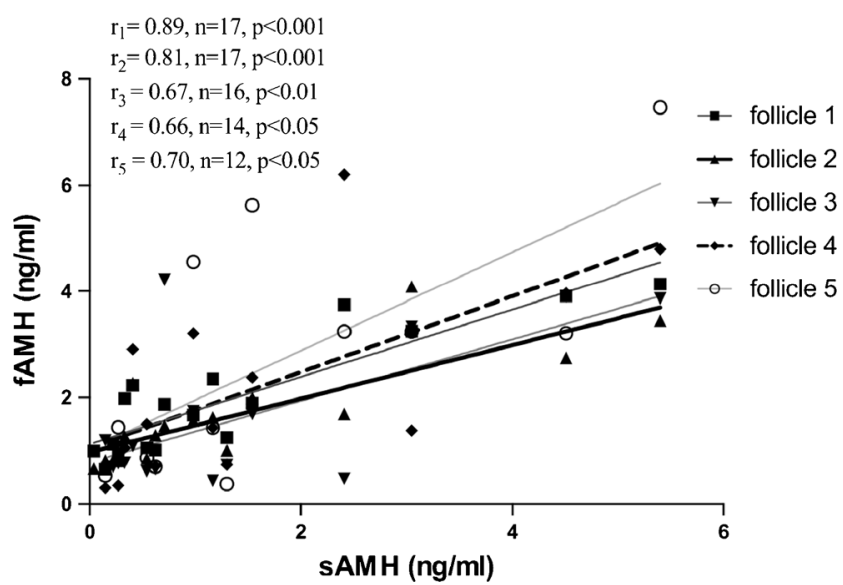

Fig. 2 Correlation of SAMH with individual fAMH values of the first five follicles of each patient

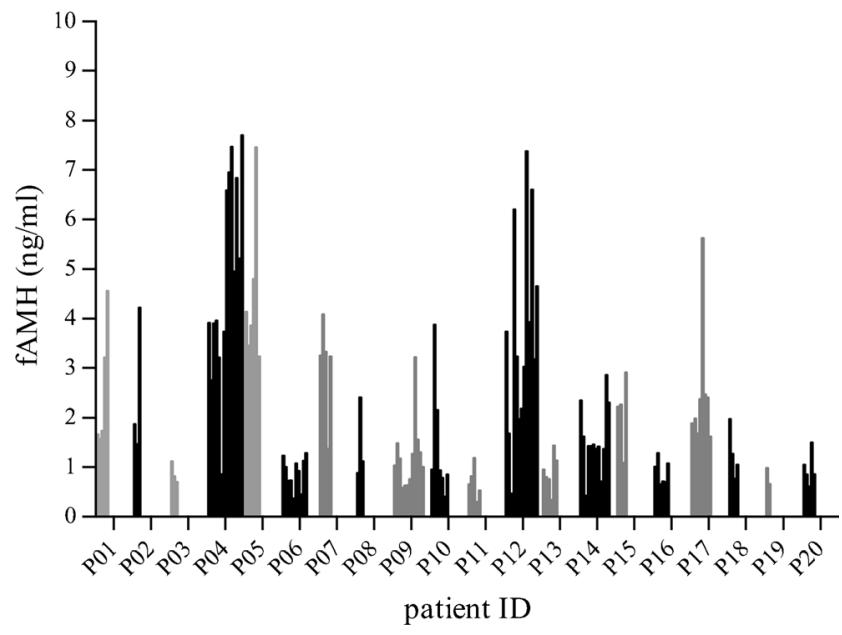

Fig. 3 Individual follicle AMH (fAMH) values in all patients (nfollicle per patient $=2-14)$. Mean fAMH concentrations significantly differed between patients (one-way ANOVA, $p<0.001$ )

correlated AMH values of the first punctured follicle [20] or pooled FF [21] with sAMH. Peripheral AMH concentrations are exclusively dependent not only on the number of follicles but also on the individual ability of each follicle to produce AMH [22]. Small follicles exhibit higher AMH concentrations compared to large ones, according to the fact that granulosa cells reduce their AMH production during final follicular maturation [23, 24]. Our results also revealed a significant difference in mean fAMH concentrations between patients, which may be based on the varying mature follicle sizes during one stimulated cycle in IVF treatment [25]. Interestingly, mean fAMH showed a trend to be negatively correlated with age, which is in line with the overall AMH decrease in patients with increasing age. Additionally, our results demonstrated decreasing sAMH concentrations with increasing age of the patient, thereby confirming the state-of-the-art opinion that sAMH values inversely correlate with age [7].

In general, fAMH concentrations are positively associated with embryo implantation when measuring the AMH concentrations in the dominant follicle [26]. Studies suggested that higher fAMH values positively correlated with fertilization [27] and implied higher chances for pregnancy [28]. On the other hand, Mehta et al. demonstrated a negative correlation between fAMH and oocyte quality, fertilization, pregnancy, and embryo implantation rates [12]. A major disadvantage of numerous studies is the diverging dataset of FF investigated. It is hardly possible to draw conclusions from pooled FF or dominant follicle analysis only. These data provided hints but did not reflect AMH concentrations in all-grown follicles available with no possibility for future association of individual FF with the respective IVF outcome. Our data provided evidence that fAMH concentrations of individual follicles of one hormonal stimulated cycle during IVF treatment did not significantly differ within one patient and resembled SAMH values. 
A limitation of the study is that we did not investigate the respective IVF outcome of different FF and associated embryos. In addition, the small sample size must be considered as a possible drawback. Another limitation is the lack of information on size and volume of the aspirated follicles, since correlation of these parameters with the IVF outcome would lead to a more conclusive analysis. However, volumetric analysis and sizing of follicles are time-consuming procedures and unnecessarily prolong anesthesia and patient discomfort.

\section{Conclusion}

In conclusion, our results clearly showed that individual fAMH concentrations reflected SAMH values of a stimulated cycle during IVF treatment and that fAMH concentrations did not significantly differ within one patient. In future studies, it will be interesting to correlate individual fAMH values to the respective embryo development and overall pregnancy outcome in order to improve IVF treatments and to refrain from embryo overproduction.

Acknowledgments Open access funding provided by Medical University of Graz. The authors want to thank the team of the Das Kinderwunsch Institut Schenk $\mathrm{GmbH}$ for their valuable help and expertise.

\section{Compliance with ethical standards}

Conflict of interest The authors declare that they have no conflict of interest.

Funding This work was supported by CBmed (Center for Biomarker Research in Medicine) with a funding of the Austrian Research Promotion Agency (FFG); project number 856,169.

Open Access This article is distributed under the terms of the Creative Commons Attribution 4.0 International License (http:// creativecommons.org/licenses/by/4.0/), which permits unrestricted use, distribution, and reproduction in any medium, provided you give appropriate credit to the original author(s) and the source, provide a link to the Creative Commons license, and indicate if changes were made.

\section{References}

1. Revelli A, Delle Piane L, Casano S, Molinari E, Massobrio M, Rinaudo P. Follicular fluid content and oocyte quality: from single biochemical markers to metabolomics. Reprod Biol Endocrinol. 2009; 7:40

2. Cate RL, Mattaliano RJ, Hession C, Tizard R, Farber NM, Cheung A, Ninfa EG, Frey AZ, Gash DJ, Chow EP. Isolation of the bovine and human genes for Müllerian inhibiting substance and expression of the human gene in animal cells. Cell. 1986;45:685-98.

3. Broer SL, Broekmans FJM, Laven JSE, Fauser BCJM. AntiMüllerian hormone: ovarian reserve testing and its potential clinical implications. Hum Reprod Update. 2014;20:688-701.
4. Carrarelli P, Rocha ALL, Belmonte G, Zupi E, Abrão MS, Arcuri F, Piomboni P, Petraglia F. Increased expression of antimüllerian hormone and its receptor in endometriosis. Fertil Steril. 2014;101: 1353-8.

5. Bhattacharya $\mathrm{P}$, Keating AF. Ovarian metabolism of xenobiotics. Exp Biol Med. 2011;236:765-71.

6. Broer SL, Mol B, Dólleman M, Fauser BC, Broekmans FJ. The role of anti-Müllerian hormone assessment in assisted reproductive technology outcome. Curr Opin Obstet Gynecol. 2010;22:193-201.

7. Lie Fong S, Visser JA, Welt CK, de Rijke YB, Eijkemans MJC, Broekmans FJ, Roes EM, Peters WHM, Hokken-Koelega ACS, Fauser BCJM, Themmen APN, de Jong FH, et al. Serum antiMüllerian hormone levels in healthy females: a nomogram ranging from infancy to adulthood. J Clin Endocrinol Metab. 2012;97: 4650-5.

8. Randolph JF, Harlow SD, Helmuth ME, Zheng H, McConnell DS. Updated assays for inhibin B and AMH provide evidence for regular episodic secretion of inhibin B but not AMH in the follicular phase of the normal menstrual cycle. Hum Reprod. 2014;29:592600 .

9. Revelli A, Biasoni V, Gennarelli G, Canosa S, Dalmasso P, Benedetto C. IVF results in patients with very low serum AMH are significantly affected by chronological age. J Assist Reprod Genet. 2016;33:603-9.

10. Tremellen K, Zander-Fox D. Serum anti-Mullerian hormone assessment of ovarian reserve and polycystic ovary syndrome status over the reproductive lifespan. Aust N Z J Obstet Gynaecol. 2015;55: 384-9.

11. Basuino L, Silveira CF. Human follicular fluid and effects on reproduction. JBRA Assist Reprod 2016; 20.

12. Mehta B, Chimote M, Chimote N, Nath N, Chimote N. Follicularfluid anti-Mullerian hormone (FF AMH) is a plausible biochemical indicator of functional viability of oocyte in conventional in vitro fertilization (IVF) cycles. J Hum Reprod Sci. 2013;6:99.

13. Tramišak Milaković T, Panić Horvat L, Čavlović K, Smiljan Severinski N, Vlašić H, Vlastelić I, Ljiljak D, Radojčić Badovinac A. Follicular fluid anti-Müllerian hormone: a predictive marker of fertilization capacity of MII oocytes. Arch Gynecol Obstet. 2015;291:681-7.

14. Kim JH, Lee JR, Chang HJ, Jee BC, Suh CS, Kim SH. AntiMüllerian hormone levels in the follicular fluid of the preovulatory follicle: a predictor for oocyte fertilization and quality of embryo. J Korean Med Sci. 2014;29:1266-70.

15. Steiner H-P. Optimizing technique in follicular aspiration and flushing. Milder, mildest or back to nature - textbook of minimal stimulation IVF. New Delhi: Jaypee Brothers Medical Publishers Ltd; 2011. p. 98-102.

16. Fauser BCJM, Diedrich K, Devroey P. Evian annual reproduction workshop group 2007. Predictors of ovarian response: progress towards individualized treatment in ovulation induction and ovarian stimulation. Hum Reprod Update. 2008;14:1-14.

17. Alviggi C, Humaidan P, Ezcurra D. Hormonal, functional and genetic biomarkers in controlled ovarian stimulation: tools for matching patients and protocols. Reprod Biol Endocrinol RBE. 2012;10:9.

18. Schenk M, Huppertz B, Obermayer-Pietsch B, Kastelic D, Hörmann-Kröpfl M, Weiss G. Biobanking of different body fluids within the frame of IVF - a standard operating procedure to improve reproductive biology research. J Assist Reprod Genet 2016.

19. Andreasson U, Perret-Liaudet A, van Waalwijk van Doorn LJC, Blennow K, Chiasserini D, Engelborghs S, Fladby T, Genc S, Kruse N, Kuiperij HB, Kulic L, Lewczuk P, et al. A practical guide to immunoassay method validation. Front Neurol. 2015;6:179.

20. Hattori Y, Sato T, Okada H, Saito C, Sugiura-Ogasawara M. Comparison of follicular fluid and serum anti-Mullerian hormone 
levels as predictors of the outcome of assisted reproductive treatment. Eur J Obstet Gynecol Reprod Biol. 2013;169:252-6.

21. Wunder DM, Guibourdenche J, Birkhäuser MH, Bersinger NA. Anti-Müllerian hormone and inhibin B as predictors of pregnancy after treatment by in vitro fertilization/intracytoplasmic sperm injection. Fertil Steril. 2008;90:2203-10.

22. Fanchin R, Louafi N, Méndez Lozano DH, Frydman N, Frydman R, Taieb J. Per-follicle measurements indicate that anti-müllerian hormone secretion is modulated by the extent of follicular development and luteinization and may reflect qualitatively the ovarian follicular status. Fertil Steril. 2005;84:167-73.

23. Baarends WM, Uilenbroek JT, Kramer P, Hoogerbrugge JW, van Leeuwen EC, Themmen AP, Grootegoed JA. Anti-müllerian hormone and anti-müllerian hormone type II receptor messenger ribonucleic acid expression in rat ovaries during postnatal development, the estrous cycle, and gonadotropin-induced follicle growth. Endocrinology. 1995;136:4951-62.

24. Vigier B, Picard J-Y, Tran D, Legeai L, Josso N. Production of antiMüllerian hormone: another homology between Sertoli and granulosa cells. Endocrinology. 1984;114:1315-20.
25. Mehri S, Levi Setti PE, Greco K, Sakkas D, Martinez G, Patrizio P. Correlation between follicular diameters and flushing versus no flushing on oocyte maturity, fertilization rate and embryo quality. J Assist Reprod Genet. 2014;31:73-7.

26. Fanchin R, Mendez Lozano DH, Frydman N, Gougeon A, di Clemente N, Frydman R, Taieb J. Anti-Müllerian hormone concentrations in the follicular fluid of the preovulatory follicle are predictive of the implantation potential of the ensuing embryo obtained by in Vitro fertilization. J Clin Endocrinol Metab. 2007;92:1796-802.

27. Aflatoonian A, Oskouian H, Ahmadi S, Oskouian L. Prediction of high ovarian response to controlled ovarian hyperstimulation: antiMüllerian hormone versus small antral follicle count (2-6 mm). J Assist Reprod Genet. 2009;26:319-25.

28. Cupisti S, Dittrich R, Mueller A, Strick R, Stiegler E, Binder H, Beckmann MW, Strissel P. Correlations between anti-müllerian hormone, inhibin B, and activin A in follicular fluid in IVF/ICSI patients for assessing the maturation and developmental potential of oocytes. Eur J Med Res. 2007;12:604-8. 